\title{
Axion-like particles and dark photons - hints from XENON1T and stellar cooling
}

\section{Felix Kahlhoefer*}

Institute for Theoretical Particle Physics and Cosmology (TTK), RWTH Aachen University, 52056 Aachen, Germany

E-mail: kahlhoefer@physik.rwth-aachen.de

The recently observed excess of electron recoil events in XENON1T can be interpreted as the absorption of bosonic dark matter particles in the $\mathrm{keV}$ range such as axion-like particles or dark photons. These same particles can also be produced in astrophysical systems such as horizontal branch stars or white dwarfs and account for the anomalous cooling rates observed in these systems. This talk considers the possibility that these phenomena have a common origin and discusses whether such an interpretation is preferred over the background hypothesis from a statistical point of view.

40th International Conference on High Energy physics - ICHEP2020

July 28 - August 6, 2020

Prague, Czech Republic (virtual meeting)

${ }^{*}$ Speaker 


\section{Introduction}

Direct detection experiments were originally conceived and designed to search for nuclear recoils from the scattering of weakly-interacting dark matter (DM) particles with masses in the $\mathrm{GeV}-\mathrm{TeV}$ range. Nevertheless, thanks to impressive achievements in terms of the background rates and the low-energy threshold, these experiments are also sensitive to any type of new physics that induces electron recoils in the $\mathrm{keV}$ range. The XENON Collaboration has recently performed such a search and observed an excess over known backgrounds in electronic recoil events with energies in the range 1-7 keV [1]. While this excess cannot be explained by varying the rates of known background, it can largely be accounted for by an unexpected tritium contamination in the detector. In spite of this plausible explanation in terms of known physics, it is exciting to consider alternative interpretations that would imply evidence for physics beyond the Standard Model.

Indeed, following the announcement from the XENON Collaboration, a large number of such interpretations have been put forward. These interpretations can broadly be divided into two categories. In the first category the XENON1T excess is explained in terms of particles produced in the sun that subsequently deposit their energy in the detector through scattering or absorption. Examples for such models include QCD axions [2] and neutrinos with non-standard interactions [3]. While these models have the attractive feature that they require comparably few new parameters in order to fit the excess, they suffer from a significant problem: In order to yield observable signals, the required production cross sections would need to be so large that these particles would constitute a non-negligible source of cooling and modify the stellar evolution in conflict with observations [2].

Models in the second category therefore consider the exciting alternative that the XENON1T excess is caused by non-relativistic particles bound to the Milky Way, which could constitute some or all of the local dark matter density. ${ }^{1}$ The simplest possibility, namely that the signal is due to the elastic scattering of $\mathrm{GeV}$-scale dark matter particles on electrons, does however not give a good fit to data [4]. Inelastic scattering on either electrons or nucleons can explain the observed excess at the cost of introducing additional parameters [4].

A particularly appealing explanation is that the XENON1T excess is caused by the absorption of keV-scale bosonic dark matter particles, such as axion-like particles (ALPs) [5] or hidden photons [6], which would convert their entire rest mass into electronic recoil energy. Although such particles would be too light and too weakly coupled to be produced thermally in the early universe, they can be produced through a number of well-known non-thermal processes, such as the misalignment mechanism. Interestingly, such keV-scale dark matter particles can also be produced in astrophysical objects and would again constitute a new cooling mechanism [7]. However, in contrast to models in the first category, the required production cross section is typically small enough to be consistent with astrophysical observations. Nevertheless, the additional cooling contribution may be non-negligible and could potentially address slight discrepancies between predicted cooling rates and observations of horizontal branch (HB) stars [8, 9] and white dwarfs (WDs) [10].

${ }^{1}$ Further proposals that do not fall into either of these two categories include dark matter particles boosted to semirelativistic velocities through astrophysical processes and relativistic particles produced in the annihilation or decay of dark matter particles. 


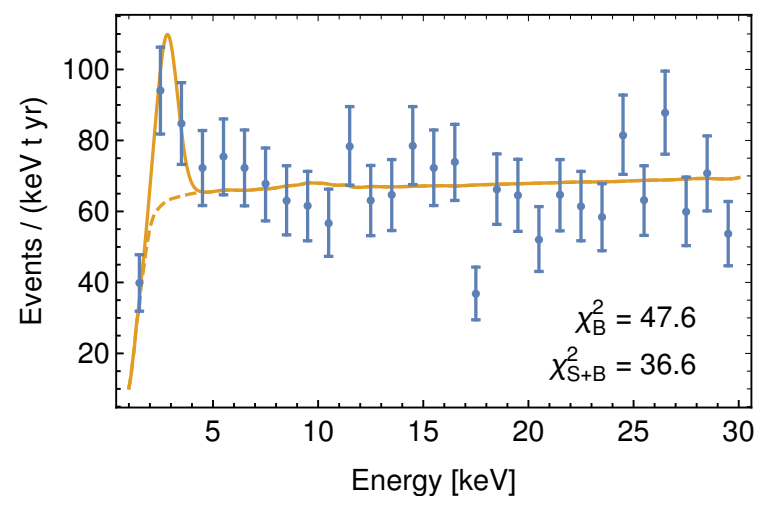

Figure 1: Predictions of the best-fitting hidden photon model compared to the observed XENON1T data (blue points and error bars). The background-only predictions are indicated by the dashed line. Figure taken from Ref. [6].

\section{Hints from XENON1T and stellar cooling}

Figure 1 shows the best-fit interpretation of the XENON1T excess in terms of dark photon absorption. The position and height of the peak are set by the dark photon mass and the kinetic mixing parameter, respectively, which are found to be $m_{X}=2.8 \mathrm{keV}$ and $\epsilon=8.6 \times 10^{-16}$ at the best-fit point [6]. The width of the peak is determined by the detector resolution. The preference for the signal+background hypothesis over the background-only hypothesis is at the level of $\Delta \chi^{2} \approx 11$. Similar values are obtained from an unbinned likelihood analysis [4] and when allowing for variations in the background normalisation [11]. The corresponding results for ALPs are shown in figure 2. The best-fit point corresponds to an ALP mass of $m_{a}=2.7 \mathrm{keV}$ and an ALP-electron coupling of $g_{a e}=5 \times 10^{-14}$ when assuming the ALPs to constitute all of dark matter. Note that ALP-photon couplings are assumed to be irrelevant for the phenomenology [5].

At first sight, the coupling strengths implied by the XENON1T excess are too small for these particles to give a relevant contribution to stellar cooling. There are, however, two important exceptions: The production of hidden photons in stellar cores receives a resonant enhancement when the plasma frequency (i.e. the mass of the visible photon) is comparable to the hidden photon mass [7]. Intriguingly, this turns out to be the case for HB stars, which are therefore particularly sensitive to hidden photons with masses of a few $\mathrm{keV}$. Second, if the particles responsible for the XENON1T excess constitute only a subdominant fraction of the local dark matter density, larger couplings are required to reproduce the observed signal. In this case ALP production rates in stars would be enhanced and can lead to relevant effects [5].

These effects are of great interest because of the so-called cooling hints: The $R$ parameter, which quantifies the ratio of HB and Red Giant Branch stars and serves as a measure for the cooling of HB stars, is slightly smaller than expected, indicating an exotic cooling mechanism [8, 9]. Likewise, several WDs exhibit a more rapid decrease in their pulsation period than expected, which is again consistent with an additional source of cooling [10]. In the following we will show that the former cooling hint can be addressed if the XENON1T excess is due to the absorption of hidden photons, while both hints can be addressd if the excess is due to a sub-dominant component of ALPs. This is indicated in the right panel of figure 2, where the blue stars indicate the improved fit 

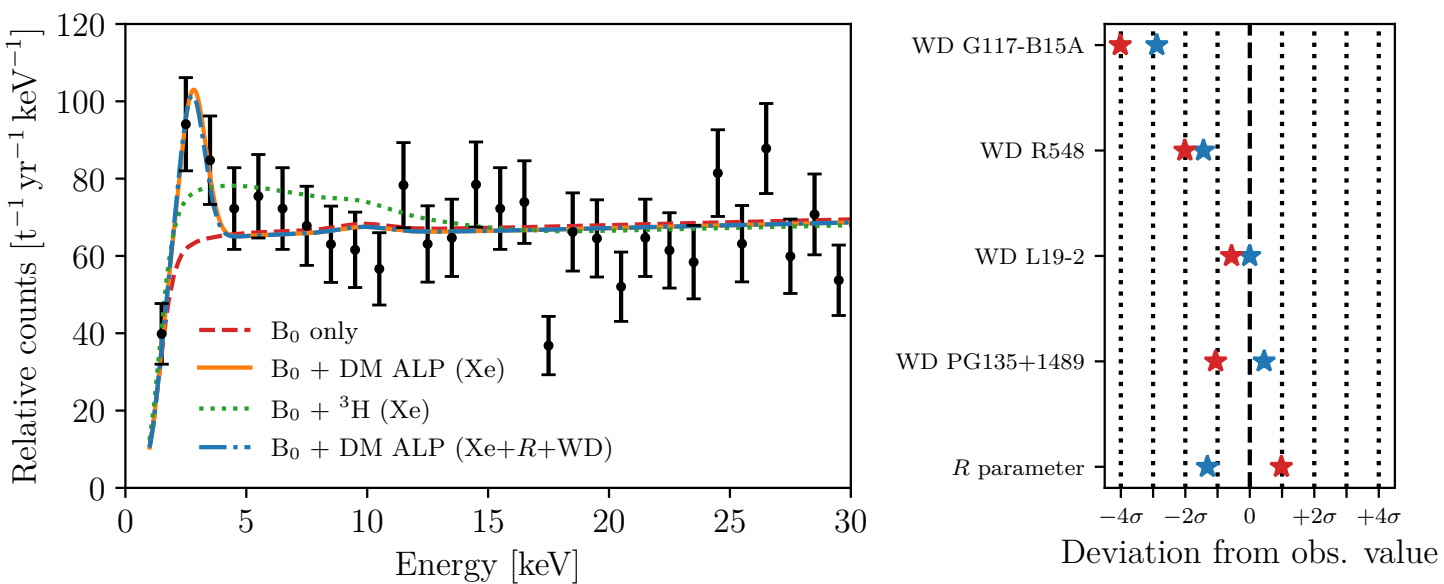

Figure 2: Predictions of the best-fitting ALP model. The left panel shows the observed XENON1T data (black points and error bars) compared to the best-fit background (dashed red), background+tritium (dotted green), and signal+background (solid orange). The ALP model giving the best fit to both XENON1T and astrophysical data is represented by a dashed-dotted blue line. The right panel shows the corresponding bestfit predictions for the WD period decrease and $R$ parameter for no ALP (red stars) and including ALPs (blue stars). Figure taken from Ref. [11].

of various astrophysical observations at the joint best-fit point.

\section{Global interpretation}

Figure 3 shows the result of a combined fit of the XENON1T excess and the $R$ parameter likelihood in the context of the hidden photon model. Clearly, the same parameter region that gives a good fit to the XENON1T excess can also potentially accommodate the preference for additional cooling from the $R$ parameter. We emphasize, however, that the cooling hint from HB stars is not very significant and that the $R$ parameter likelihood does not account for the fact that different HB stars will have slightly different plasma frequencies. Our results therefore need to be interpreted with care (see Ref. [6] for further discussion).

Figure 4 shows the corresponding results for the case of ALPs. The left (right) panel shows the best-fit contours for the ALP-electron coupling and the ALP fraction of the local dark matter density $\eta$ (the ALP mass). For a sub-dominant fraction of ALPs $(\eta \approx 0.1)$ the required ALP-electron couplings are sufficiently large to simultaneously address the cooling hints from HB stars and WDs. Because the latter are quite significant, the preference of the signal+background hypothesis compared to the background-only hypothesis grows to $\Delta \chi^{2}=23.1$ and is still considerable when allowing for an additional tritium contribution $\left(\Delta \chi^{2}=14.9\right)$ [11].

While these numbers look rather impressive, they need to be interpreted with care. A purely frequentist interpretation is challenging, as Wilks' theorem may be violated and the look-elsewhere effect needs to be taken into account. It is therefore interesting to complement the analysis presented above by a Bayesian interpretation, which automatically includes an Occam penalty for models that make very unspecific predictions. The drawback is that the results of such an analysis are typically sensitive to the priors, which encode our previous knowledge about the model. 


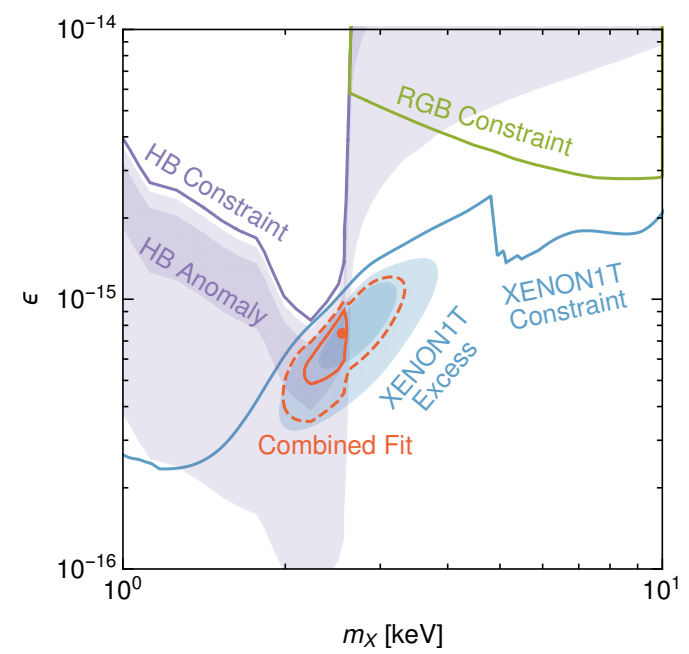

Figure 3: Region in the hidden photon parameter space hinted at by the XENON1T result [1] (blue regions showing $68 \%$ and $95 \%$ C.L. contours) compared to the regions suggested from stellar cooling [8, 9] (purple regions). The corresponding lines indicate 95\% C.L. exclusion limits, while the green line represents the exclusion limit from RGB stars. The red lines show the preferred parameter regions from a combined fit of the XENON1T excess and the HB anomaly at $68 \%$ and $95 \%$ C.L. The best-fit point is indicated by a red dot. Figure taken from Ref. [6].

When adopting very broad priors for the ALP mass, relic density and coupling strength, we find that Bayesian model comparison reveals no clear preference for the signal+background model over the background-only hypothesis (see Ref. [11] for details). This reflects the fact that, while the ALP model can accommodate the XENON1T excess, it can predict neither the magnitude nor the position of the signal a priori. However, when considering more narrow priors (for example by including astrophysical bounds as prior knowledge and calculating partial Bayes factors) the preference for the ALP model grows, although it never reaches the level of substantial evidence.

\section{Outlook}

What makes the XENON1T excess so intriguing is that it can be tested by the next generation of direct detection experiments. If the signal is confirmed, the next step will be to investigate whether it is indeed due to the absorption of $\mathrm{keV}$-scale bosonic dark matter particles.

A promising strategy for this purpose would be to map out the time dependence of the signal. In contrast to most alternative explanations of the excess, the absorption signal does not typically exhibit an annual modulation. However, time-dependent signals may arise from small-scale inhomogeneities in the dark matter densitiy, which are a generic prediction of hidden photon and ALP models. Whenever the Earth crosses such a local overdensity the expected event rate would be boosted substantially, leading to a non-trivial correlation between observed events.

At the same time, more detailed observations and an improved modelling of stellar processes will be essential to understand whether there is indeed a preference for exotic cooling mechanisms. If this turns out to be the case, a joint explanation of these hints and the XENON1T excess in terms of hidden photons or ALPs is an exciting possibility. 

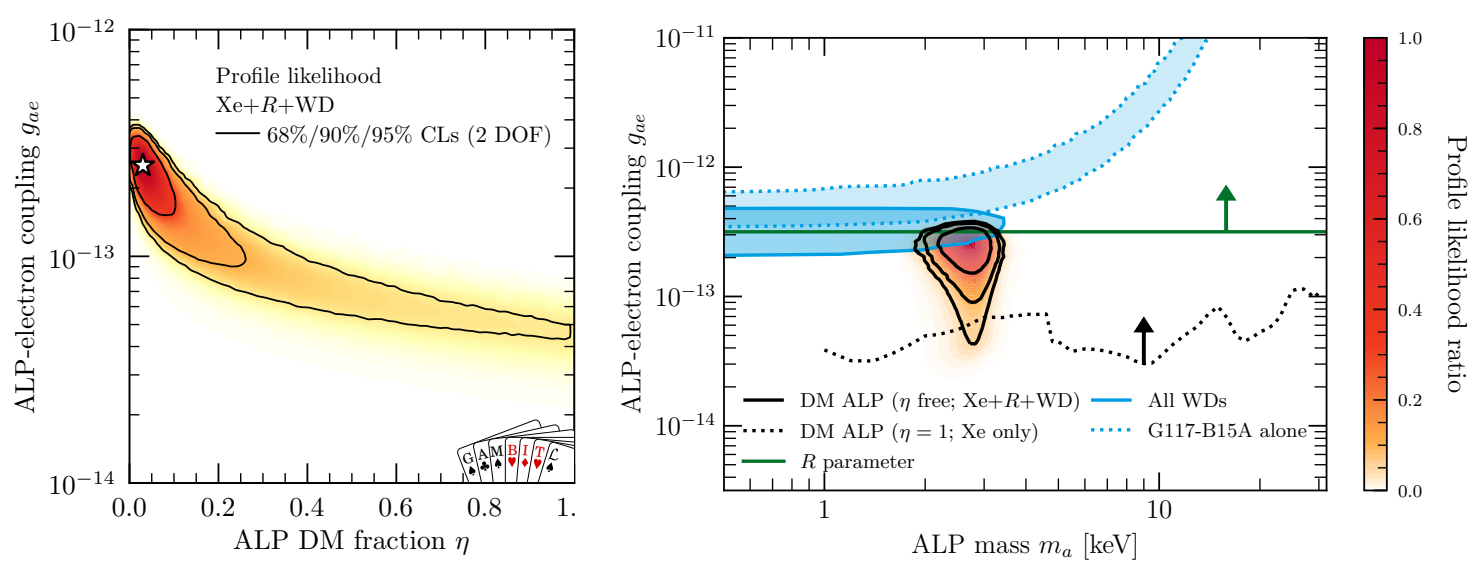

Figure 4: The profile likelihood obtained by combining the XENON1T excess with the $R$ parameter and WD data. The star in the left panel indicates the best-fit point. For context, we show in the right panel the 90\% CL constraints from XENON1T assuming $\eta=1$ (dotted black), the $R$ parameter (green), and the region hinted by a combination of WDs (shaded blue region, solid line). We also show one WD (G117-B15A) alone for comparison (shaded blue region, dotted line). The arrows point towards the excluded regions. Figure taken from Ref. [11].

\section{References}

[1] XENON, E. Aprile et al., Phys. Rev. D 102, 072004 (2020), 2006.09721.

[2] L. Di Luzio, M. Fedele, M. Giannotti, F. Mescia, and E. Nardi, Phys. Rev. Lett. 125, 131804 (2020), 2006.12487.

[3] C. Boehm, D. G. Cerdeno, M. Fairbairn, P. A. Machado, and A. C. Vincent, (2020), 2006.11250 .

[4] I. M. Bloch et al., (2020), 2006.14521.

[5] F. Takahashi, M. Yamada, and W. Yin, Phys. Rev. Lett. 125, 161801 (2020), 2006.10035.

[6] G. Alonso-Álvarez, F. Ertas, J. Jaeckel, F. Kahlhoefer, and L. J. Thormaehlen, JCAP 11, 029 (2020), 2006.11243.

[7] H. An, M. Pospelov, J. Pradler, and A. Ritz, Phys. Lett. B 747, 331 (2015), 1412.8378.

[8] A. Ayala, I. Domínguez, M. Giannotti, A. Mirizzi, and O. Straniero, Phys. Rev. Lett. 113, 191302 (2014), 1406.6053.

[9] M. Giannotti, I. Irastorza, J. Redondo, and A. Ringwald, JCAP 05, 057 (2016), 1512.08108.

[10] S. Hoof, F. Kahlhoefer, P. Scott, C. Weniger, and M. White, JHEP 03, 191 (2019), 1810.07192, [Erratum: JHEP 11, 099 (2019)].

[11] P. Athron et al., (2020), 2007.05517. 